\title{
Rendimiento cognitivo en mujeres con síndrome de ovario poliquístico: un estudio piloto*
}

\author{
Cognitive Performance in Women with Polycystic Ovarian \\ Syndrome: A Pilot Study
}

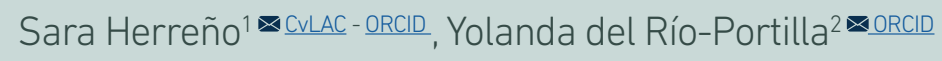

1 Universidad Pontificia Bolivariana, Colombia.

12 Universidad Nacional Autónoma de México, México.

Fecha correspondencia:

Recibido: septiembre 7 de 2017.

Aceptado: diciembre 14 de 2018.

Forma de citar:

Herreño, S., \& del Río-Portilla, Y.

(2019). Rendimiento cognitivo en

mujeres con Síndrome de Ovario

Poliquístico: un estudio piloto. Rev.

CES PSico, 12(2), 41-50.

Open access

(c) Copyright

Licencia creative commons

Ética de publicaciones

Revisión por pares

Gestión por Open Journal System

DOl: http://dx.doi.org/10.21615/

cesp.12.2.4

ISSN: 2011-3080

Sobre el artículo:

* Fuentes de financiación: financiado parcialmente por DGAPA (proyecto IN228409) y la Coordinación del Programa de Maestría y Doctorado en Psicología

Comparte

\section{Resumen}

El Síndrome de ovario poliquístico (SOP) es la enfermedad endocrina-metabólica más frecuente en las mujeres en edad reproductiva. A pesar de su alta prevalencia, hay pocas investigaciones que analizan los efectos de los cambios hormonales sobre la cognición de pacientes con SOP. Objetivo: comparar el rendimiento en habilidades cognitivas y los niveles de hormonas sexuales de un grupo de pacientes con SOP y con las de un grupo control. Método: Participaron 20 mujeres mayores de 21 años, sin tratamiento hormonal, dividas en dos grupos, el primero compuesto por 10 pacientes diagnosticadas con SOP según los criterios de Rotterdam (GSOP) y el otro, de control, compuesto por 10 mujeres sin diagnóstico de SOP (GCT). Se aplicaron las pruebas WAIS III y fluidez verbal semántica y fonológica de la batería NEUROPSI Atención y memoria, y se realizaron análisis sanguíneos de hormonas sexuales. Resultados: Las pacientes del GSOP obtuvieron puntajes inferiores a las del GCT en el CI Verbal ( $p=0.009)$, Cl Total ( $p=0.029)$, índice de comprensión verbal $(p=0.005)$, índice de memoria de trabajo $(p=0.023)$ y en la prueba de fluidez verbal semántica $(p=0.029)$. No se encontraron diferencias significativas en los niveles hormonales. Conclusión: el GSOP presentó menor rendimiento que el GCT en pruebas de tipo verbal, aunque no se presentó déficit en su ejecución. Se sugiere estudiar la relación de la insulinorresistencia con la cognición en pacientes con SOP.

Palabras clave: Síndrome de Ovario Poliquístico (SOP), Hormonas Gonadales, Hormonas Sexuales, Cognición. Funcionamiento Cognitivo.

\section{Abstract}

Polycystic ovarian syndrome (PCOS) is the most common endocrine-metabolic disease in women of reproductive age. Despite its high prevalence, little research analyzes the effects of hormonal changes on cognition in patients with PCOS. Objective: To compare the performance in cognitive abilities and sexual hormone levels of a group of patients with PCOS and those of a control group. Method: Twenty women older than 21 years, without hormone treatment, divided in two groups, the first consisting by 10 patients diag- 
de la Universidad Nacional Autónoma de México. Este artículo presenta resultados parciales de la tesis doctoral de Sara Herreño, quien fue beneficiara del acuerdo 206 de la Universidad Pontificia Bolivariana para realizar sus estudios doctorales, radicado CIDI 851B-07/17-63.

Agradecimientos: Las autoras agradecen a la Dra. Elsa Díaz López y a la Dra. Leticia Bedoya (Grupo Especializado en Salud Femenina - GESFEM) su colaboración para el diagnóstico y la selección de las participantes; y a la Lic. María del Carmen Castro González por su colaboración en la aplicación de pruebas psicológicas.

\section{Sobre los autores:}

1. Estudiante de Doctorado de Psicología. Magister en Ciencias del Comportamiento. Psicóloga. Docente investigadora Laboratorio de sueño, Facultad de Psicología, Universidad Nacional Autónoma de México, México; y Facultad de Psicología, Universidad Pontificia Bolivariana, Medellín, Colombia.

2. Doctora en Psicología. Magister en Psicobiología. Psicóloga. Docente Investigadora Laboratorio de sueño y Coordinación de Psicobiología y Neurociencias, Facultad de Psicología, Universidad Nacional Autónoma de México, México. nosed with PCOS according to the Rotterdam criteria (GSOP), and the other composed of 10 women without diagnosis of PCOS (GCT). WAIS III and semantic and phonological verbal fluency tests of the NEUROPSI attention and memory test battery were applied, and blood analyzes of sexual hormones were analyzed. Results: The GSOP patients obtained lower scores than the GCT in Verbal IQ ( $p=0.009)$, full scale IQ $(p=0.029)$, verbal comprehension index ( $p=0.005)$, working memory index $(p=0.023)$ and semantic verbal fluency test $(p=0.029)$. No significant differences were found in sexual hormone levels. Conclusion: the GSOP showed lower performance than the GCT in verbal tests, although there was not deficit in its execution. It is suggested to study the relationship of insulin resistance with cognition in patients with GSOP.

Keywords: Polycystic Ovary Syndrome (PCOS), Gonadal Hormones, Sexual Hormones, Cognition, Cognitive Functioning.

\section{Introducción}

El Síndrome de Ovario Poliquístico (SOP) constituye la endocrinopatía más frecuente en las mujeres en edad reproductiva presentando una prevalencia entre el 6 al 10\% (Bozdag, Mumusoglu, Zengin, Karabulut, \& Yildiz, 2016). Particularmente, en México se ha reportado una prevalencia entre 6\% y 6.6\% (Moran et al., 2010).

Según los criterios de Rotterdam, para diagnosticar el SOP se requiere que la paciente presente al menos dos de los siguientes síntomas: oligo/anovulación relacionada con ciclos menstruales irregulares o amenorrea; signos clínicos y/o bioquímicos de hiperandrogenismo (acné, hirsutismo, alopecia y/o exceso de testosterona o testosterona libre); y ovarios de apariencia ecográfica poliquística (Rotterdam ESHRE/ASRM-Sponsored PCOS Consensus Workshop Group, 2004). Además, se ha observado que estas pacientes podrían presentar síntomas metabólicos como insulinorresistencia, obesidad, dislipidemias, entre otras (Anagnostis, Tarlatzis, \& Kauffman, 2018).

A pesar de su alta prevalencia, existen pocas investigaciones que analizan los efectos de los cambios hormonales, como el hiperandrogenismo, sobre la cognición en pacientes con SOP (Martino, Bonet, \& De Bortoli, 2015). Schattmann y Sherwin (2007a) relacionaron el nivel de testosterona libre presente en pacientes con SOP y la ejecución de diversas tareas cognitivas, y encontraron que las mujeres con SOP tuvieron menor desempeño que el grupo de control en pruebas de memoria de trabajo visoespacial, fluidez verbal y destreza motora; lo cual indicaría un efecto negativo de la testosterona sobre el rendimiento cognitivo en estas pacientes.

Por su parte, Barnard, Balen, Ferriday, Tiplady y Dye (2007) hallaron que un grupo de pacientes con SOP presentó mayor tiempo de reacción y número de errores en una prueba de atención en comparación con un grupo de control, pero no encontraron diferencias entre grupos en tareas de rotación mental y localización espacial. No obstante, en un estudio posterior Barry, Parekh y Hardiman (2013) reportaron que las mujeres con SOP presentaron mejor desempeño en una prueba de rotación mental en tres dimensiones respecto al grupo de control, y señalaron que estos resultados correlacionaron positivamente con los niveles de testosterona y negativamente con los niveles de estrógeno en las pacientes con SOP.

En un estudio reciente, Rees, Udiawar, Berlot, Jones y O'Sullivan (2016) evaluaron a pacientes con SOP en una serie de pruebas cognitivas encontrando que presentaron menor rendimiento en pruebas de memoria a corto plazo, memoria de trabajo, voca- 
Pág 43

Schattmann y Sherwin (2007a) relacionaron el nivel de testosterona libre presente en pacientes con SOP y la ejecución de diversas tareas cognitivas, y encontraron que las mujeres con SOP tuvieron menor desempeño que el grupo de control en pruebas de memoria de trabajo visoespacial, fluidez verbal y destreza motora; lo cual indicaría un efecto negativo de la testosterona sobre el rendimiento cognitivo en estas pacientes. bulario, semejanzas y memoria episódica, en comparación con un grupo de control; sin embargo, señalan que estos resultados no correlacionaron con el nivel de testosterona o de insulina.

Tomando en cuenta estos antecedentes, es posible hipotetizar que las pacientes diagnosticadas con SOP podrían presentar un menor puntaje en pruebas verbales que las mujeres sin este diagnóstico y estos puntajes podrían estar asociados con las características hormonales presentes en las pacientes con SOP. Sin embargo, hasta la fecha, son pocos los estudios que abordan esta problemática por lo que se requiere mayor evidencia para apoyar esta hipótesis.

Dado lo anterior, el objetivo del estudio es comparar el rendimiento en habilidades cognitivas, evaluadas a través de la prueba de Inteligencia Wechsler para Adultos (WAIS III) y de la prueba de fluidez verbal, y los niveles de hormonas sexuales, de un grupo de pacientes con SOP con las de un grupo de control.

\section{Método}

Estudio analítico transversal con diseño de casos/controles.

\section{Participantes}

Mediante un muestreo por conveniencia se seleccionó un grupo de mujeres con diagnóstico de SOP (GSOP) (n=10), con un promedio de edad de 26,1 años y 15,5 años de escolaridad. El diagnóstico fue realizado por un equipo de especialistas en salud de la mujer siguiendo los criterios de Rotterdam ESHRE/ASRM-Sponsored PCOS Consensus Workshop Group (2004), los cuales incluyen dos o más de los siguientes síntomas: oligo/amenorrea, evidencia clínica y/o bioquímica de hiperandrogenismo (hirsutismo, acné y/o alopecia) y presencia de ovarios poliquísticos. Y un grupo control (GCT) $(n=10)$ conformado por mujeres con ciclos menstruales regulares (ciclos de 28-35 días), con un promedio de edad de 26,2 años y 16,3 años de escolaridad.

Los criterios de inclusión para participar en el estudio fueron: tener una escolaridad mínima de 12 años y ser mayor de 21 años. Y los criterios de exclusión fueron: estar en embarazo; tomar sustancias que alteren el sistema nervioso central; presentar niveles hormonales fuera del rango normal que indiquen hiper o hipo tiroidismo, hiperprolactinemia, y/o hiperplasia suprarrenal; padecer enfermedades neurológicas y/o psiquiátricas; tener antecedentes de lesión o traumatismo cerebral; y obtener un puntaje inferior a 90 en la prueba Wechsler de Inteligencia para adultos (WAIS III). Y para las participantes del GSOP, utilizar anticonceptivos hormonales u otro tratamiento para el SOP.

\section{Instrumentos}

Escala Wechsler de inteligencia para adultos (WAIS - III, abreviatura de Wechsler Abbreviated Scale of Intelligence), versión estandarizada para México (Wechsler, 1997/2003a). Esta prueba permite obtener el cociente intelectual (CI) Verbal, Cl de Ejecución y Cl Total. Además, ofrece puntuaciones que reflejan el funcionamiento intelectual en cuatro escalas índice: Comprensión verbal (ICV), Organización perceptual (IOP), Memoria de trabajo (IMT) y Velocidad de procesamiento (IVP). La media de las puntuaciones normalizadas $\mathrm{Cl}$ e índices es 100, con desviación estándar (DS) de 15. Los coeficientes de confiabilidad de las subpruebas del WAIS III se encuentran entre 0.82 a 0.93 y para las escalas de Cl e índices oscilan entre 0.88 a 0.97 (Wechsler, 1997/2003b). 
Escala de Fluidez verbal semántica y fonológica de la batería NEUROPSI Atención y memoria (Ostrosky-Solís et al., 2003). Esta prueba es utilizada para evaluar lenguaje y funciones ejecutivas. La modalidad fonológica se evaluó solicitando a las participantes que nombraran en un minuto la mayor cantidad de palabras que iniciaban con la letra P, evitando mencionar nombres propios y palabras derivadas; y en la prueba de fluidez verbal semántica se solicitó a las participantes que nombraran, en un minuto, todos los elementos de la categoría de animales que pueda (Ostrosky-Solís et al., 2003). La batería NEUROPSI atención y memoria está compuesta por pruebas que han sido adaptadas y validadas en México tomando en cuenta la edad y escolaridad del evaluado (Ostrosky-Solís et al., 2003); las cuales se utilizaron en la presente investigación. Respecto a la prueba de fluidez verbal se reporta que la media de palabras recuperadas en la modalidad semántica para la población de 16 a 30 años con 10-22 años de escolaridad es de 23.5 con DS de 4.8 y para la modalidad fonológica la media es 16.4 con DS de 4.7 (Ostrosky-Solís et al., 2007).

\section{Procedimiento}

Las participantes con diagnóstico de SOP fueron remitidas por médicos especialistas luego de haber sido diagnosticadas y, posteriormente, invitadas participar en el estudio. Por su parte, las participantes del grupo de control se captaron mediante convocatoria realizada en el campus de la Universidad Nacional Autónoma de México, mediante carteles y redes sociales; y se seleccionaron aquellas que presentaran similar edad y nivel educativo que el grupo SOP.

En el primer contacto con las participantes voluntarias se les explicó el proceso que conllevaba su participación y se realizó una anamnesis para determinar si cumplían con los criterios de inclusión y exclusión establecidos para el estudio. Posteriormente, y previa lectura y aprobación del consentimiento informado por parte de las participantes, se aplicó la prueba WAIS III. Las pruebas de fluidez verbal semántica y fonológica del NEUROPSI se adelantaron de manera individual durante la fase folicular del ciclo menstrual (días 3 al 9 del ciclo) de cada una de las participantes; de igual manera, las muestras de sangre para los análisis hormonales fueron tomadas durante esta fase'. Se realizaron análisis sanguíneos de: hormona luteinizante (LH), hormona folículo estimulante (FSH), estradiol, progesterona, prolactina, testosterona y testosterona libre, además se realizaron las pruebas glucosa, TSH, T3, T4, 17-hidroxiprogesterona y de prolactina para considerar los criterios de exclusión. Las muestras fueron tomadas y procesadas por un laboratorio clínico especializado.

\section{Consideraciones éticas}

El estudio siguió los principios bioéticos de autonomía, beneficencia, no maleficencia y justicia. Las participantes firmaron el respectivo consentimiento informado mediante el cual se les explicó el objetivo de la investigación, en qué consistía la participación, los beneficios y posibles riesgos, el manejo confidencial de la información y la libertad de poderse retirar del estudio, además se otorgó un espacio para aclarar sus dudas. Además, se otorgó a las participantes un apoyo equivalente a 20 USD para ser utilizados en su transporte a las diferentes sesiones de evaluación y toma de muestras sanguíneas.

\section{Análisis de datos}

Se utilizó la prueba $U$ de Mann-Whitney para comparar los resultados de los dos grupos en las pruebas WAIS III: Cl total, Cl verbal, Cl de ejecución, índice de comprensión verbal, índice de memoria de trabajo, índice de organización perceptual e 
índice de velocidad de procesamiento, fluidez verbal fonológica y semántica, y para los resultados hormonales LH, FSH, estradiol, progesterona, prolactina, testosterona y testosterona libre. Se efectuó un análisis de componentes principales y rotación Varimax para conocer si las variables covarían formando un conjunto o componente (Eigenvector) con variables relacionadas entre sí e independientes de los demás conjuntos o componentes (Jolliffe, 1986) y, posteriormente, se realizó la prueba U de Mann-Whitney para conocer si existían diferencias entre los dos grupos en los componentes formados.

\section{Resultados}

Debido a la aplicación de los criterios de exclusión que involucraron niveles hormonales (prolactina, TSH y 17-Hidroxiprogesterona) fuera del rango normal esperado y otros tratamientos para el SOP, se excluyeron del estudio los datos de siete participantes. La muestra final quedó conformada por 10 participantes en el grupo SOP y 10 participantes en el grupo de control.

En la prueba WAIS III, los dos grupos presentaron puntuaciones de CI Total dentro del rango normal (igual o superior a 90), sin embargo, se encontró que las participantes del GSOP obtuvieron puntajes inferiores al GCT en el $\mathrm{Cl}$ Verbal $(U=16.5, p=0.009), \mathrm{Cl}$ Total $(U=21, p=0.029)$, el ICV $(U=14, p=0.005)$ y el IMT $(U=20.50, p=0.023)$ (Tabla 1).

En la prueba de fluidez verbal semántica y fonológica del NEUROPSI, los dos grupos presentaron un rendimiento similar al esperado para su edad y escolaridad (fluidez semántica media=23.5, DS= 4.8 y fluidez fonológica media=16.4, DS=4.7); las puntuaciones del GCT presentaron una mediana en la escala de fluidez semántica de 22 palabras y de 19 en la fonológica, mientras que la mediana de las puntuaciones del GSOP fue de 20.5 palabras en la prueba semántica y 16 en la a fonológica. No obstante, el GSOP presentó menor rendimiento en la prueba de fluidez semántica que el GCT $(U=21.5, p=0.029)$ (Tabla1).

Tabla 1. Resultados de las pruebas cognitivas para los grupos de control y grupo con diagnóstico de Síndrome de Ovario Poliquístico (SOP)

\begin{tabular}{lccccc}
\hline Variables & $\begin{array}{c}\text { Rango promedio } \\
\text { Control }\end{array}$ & $\begin{array}{c}\text { Rango } \\
\text { promedio SOP }\end{array}$ & $\boldsymbol{U}$ de Mann-Whitney & $\boldsymbol{Z}$ & Sig. (p) \\
\hline Cl verbal & 13.85 & 7.15 & 16.5 & -2.539 & $\mathbf{0 . 0 0 9 *}^{*}$ \\
\hline Cl de ejecución & 11.30 & 9.70 & 42 & -.609 & 0.579 \\
\hline $\boldsymbol{C I}$ Total & 13.40 & 7.60 & 21 & -2.195 & $\mathbf{0 . 0 2 9}^{*}$ \\
\hline ICV & 14.10 & 6.90 & 14 & -2.731 & $\mathbf{0 . 0 0 5}^{*}$ \\
\hline IOP & 11.15 & 9.85 & 43.5 & -.494 & 0.631 \\
\hline IMT & 13.45 & 7.55 & 20.5 & -2.279 & $\mathbf{0 . 0 2 3}^{*}$ \\
\hline IVP & 12.05 & 8.95 & 34.5 & -1.177 & 0.247 \\
\hline Fluidez fonológica & 11.70 & 9.30 & 38 & -.911 & 0.393 \\
\hline Fluidez semántica & 13.35 & 7.65 & 21.5 & -2.183 & $\mathbf{0 . 0 2 9 ^ { * }}$ \\
\hline
\end{tabular}

Nota: ICV: índice de comprensión verbal, IOP: índice de organización perceptual, IMT: índice de memoria de trabajo, IVP: índice de velocidad de procesamiento. ${ }^{*} \mathrm{p} \leq .05$

En cuanto al perfil hormonal, se utilizaron los intervalos de referencia para la fase folicular de las hormonas LH: 2.58-12.1 mUI/mL, FSH: 1.98-11.6 mUI/mL, Progesterona: $0.14-2.03 \mathrm{ng} / \mathrm{mL}$ y Estradiol: $26.6-161 \mathrm{pg} / \mathrm{mL}$, y los intervalos de referencia para mujeres en las pruebas de Prolactina: $3.0-18.6 \mathrm{ng} / \mathrm{mL}$, Testosterona: $5.71-77 \mathrm{ng} / \mathrm{dL}$ y 
Testosterona libre: $<2.57 \mathrm{pg} / \mathrm{mL}$. No se encontraron diferencias significativas en los niveles hormonales entre los dos grupos (Tabla 2).

Tabla 2. Perfil hormonal para los grupos de control y grupo con diagnóstico de Síndrome de Ovario Poliquístico (SOP)

\begin{tabular}{lccccc}
\hline $\begin{array}{l}\text { Variables } \\
\text { (Hormonas) }\end{array}$ & $\begin{array}{c}\text { Rango promedio } \\
\text { Control }\end{array}$ & $\begin{array}{c}\text { Rango } \\
\text { promedio SOP }\end{array}$ & U de Mann-Whitney & Z & Sig. (p) \\
\hline Luteinizante & 9.70 & 11.30 & 42 & -.605 & 0.579 \\
\hline Folículo estimulante & 11.70 & 9.30 & 38 & -.907 & 0.393 \\
\hline Prolactina & 12.30 & 8.70 & 32 & -1.361 & 0.190 \\
\hline Progesterona & 8.65 & 12.35 & 31.5 & -1.4 & 0.165 \\
\hline Estradiol & 10.75 & 10.25 & 47.5 & -.189 & 0.853 \\
\hline Testosterona & 11.50 & 9.50 & 40 & -.756 & 0.481 \\
\hline Testosterona libre & 10 & 11 & 45 & -.378 & 0.739 \\
\hline
\end{tabular}

Los resultados del análisis de componentes principales (Tabla 3) explican un 78.78\% de la varianza. Este análisis mostró una agrupación en cinco componentes, siendo el factor de habilidad verbal el que explica el 19.52\% de la varianza y también en el que se presentaron diferencias significativas entre los grupos $(U=15, p=0.0091)$.

Tabla 3. Matriz de componentes principales para las pruebas WAIS-III, Fluidez verbal y análisis hormonales

\begin{tabular}{lccccc}
\hline \multicolumn{1}{c}{ Variables } & \multicolumn{5}{c}{ Componentes (Eigenvectores) } \\
& $\begin{array}{c}\text { Factor } \mathbf{1} \\
\text { Habilidad verbal }\end{array}$ & $\begin{array}{c}\text { Factor } \mathbf{2} \\
\text { Ejecución }\end{array}$ & $\begin{array}{c}\text { Factor } \mathbf{3} \\
\text { Estrategia cognitiva }\end{array}$ & $\begin{array}{c}\text { Factor } \mathbf{4} \\
\text { Hormonas sexuales }\end{array}$ & $\begin{array}{c}\text { Factor } \mathbf{5} \\
\text { Fluidez semántica }\end{array}$ \\
\hline CI Verbal & $\mathbf{0 , 8 9 4}$ & 0,278 & 0,217 & $-0,079$ & 0,139 \\
Cl Ejecución & 0,015 & $\mathbf{0 , 9 4 2}$ & 0,04 & 0,014 & 0,012 \\
CI Total & 0,521 & $\mathbf{0 , 7 8 4}$ & 0,131 & $-0,035$ & 0,096 \\
ICV & $\mathbf{0 , 7 7}$ & $-0,061$ & 0,494 & 0,012 & 0,132 \\
IOP & 0,077 & 0,513 & $\mathbf{0 , 6 8 4}$ & 0,214 & $-0,034$ \\
IMT & $\mathbf{0 , 7 5 7}$ & 0,193 & $-0,236$ & $-0,119$ & $-0,128$ \\
IVP & 0,322 & $\mathbf{0 , 7 0 2}$ & $-0,043$ & 0,041 & $-0,456$ \\
Fluidez fonológica & 0,038 & 0,047 & $\mathbf{0 , 7 9 9}$ & $-0,02$ & $-0,043$ \\
Fluidez semántica & 0,509 & 0,146 & 0,37 & $-0,305$ & $-\mathbf{0 , 5 8 1}$ \\
Luteinizante & $-0,113$ & 0,157 & 0,056 & $\mathbf{0 , 8 3 3}$ & 0,106 \\
FSH & 0,016 & 0,03 & 0,222 & 0,283 & $\mathbf{0 , 8 4 7}$ \\
Prolactina & 0,086 & $-0,018$ & $\mathbf{0 , 7 2 7}$ & 0,115 & 0,304 \\
Progesterona & $-0,312$ & $-0,053$ & 0,448 & $\mathbf{0 , 7 0 6}$ & 0,077 \\
Estradiol & $-0,199$ & 0,017 & $-0,058$ & 0,254 & $\mathbf{- 0 , 6 4 9}$ \\
Testosterona & $\mathbf{0 , 5 6 9}$ & $-0,028$ & $-0,397$ & $-0,433$ & 0,389 \\
Testosterona Libre & 0,184 & $-0,533$ & $-0,192$ & $\mathbf{0 , 6 5 1}$ & $-0,213$ \\
\hline Eigenvalues & 3.1232 & 2.7138 & 2.589 & 2.1235 & 2.0554 \\
\hline Varianza explicada & $19.52 \%$ & $16.96 \%$ & $16.18 \%$ & $13.27 \%$ & $12.85 \%$ \\
\hline Valor de p & $\mathbf{0 . 0 0 9 1 *}$ & 0.0452 & 0.1859 & 0.3075 & 0.7913 \\
\hline
\end{tabular}

Nota: Valores rotados (Varimax) del Análisis de componentes principales, en negrita se resaltan factores con valores mayores a 0.55, y eigenvalues mayores a 1 , el porcentaje de varianza explicada en cada eigenvector y nivel de significancia menor a 0.01 de la prueba U de Mann-Whitney entre grupo de control y grupo SOP. Índice de comprensión verbal (ICV). índice de organización perceptual (IOP), índice de memoria de trabajo (IMT) e índice de velocidad de procesamiento (IVP). LH: hormona luteinizante, FSH: hormona folículo estimulante. 
Pág 47

Investigaciones sugieren que las hormonas sexuales influyen en el rendimiento en pruebas cognitivas, y los resultados de la presente investigación muestran evidencia a favor de esta hipótesis, puesto que las participantes del GSOP presentaron menor puntaje en el Cl Verbal, comprensión verbal, memoria de trabajo y fluidez semántica que las del GCT.

\section{Discusión}

Los resultados muestran diferencias significativas en el rendimiento en las pruebas cognitivas entre las participantes de un grupo de con diagnóstico de SOP (GSOP) y el grupo control (GCT); específicamente, las participantes del GSOP presentaron menores puntajes en el CI Verbal y el Cl Total de la Escala Wechsler de inteligencia para adultos, las escalas ICV, IMT y la escala de fluidez verbal semántica del NEUROPSI en comparación con las del GCT, no obstante, el puntaje obtenido por las participantes del GSOP en las pruebas mencionadas se encuentra en los rangos normales esperados para su edad y escolaridad. Además, no se encontraron diferencias significativas en los perfiles hormonales entre las participantes de ambos grupos.

Diversos estudios sobre diferencias sexuales señalan que los hombres obtienen menores puntajes en pruebas verbales y mayores puntajes en pruebas de rotación mental en relación con las mujeres (Corsi-Cabrera, del Río- Portilla, \& Muñoz-Torres, 2007; Scheuringer \& Pletzer, 2017; Voyer, 2011); lo que señala un posible efecto de los esteroides sexuales sobre el desarrollo y el posterior funcionamiento cerebral (Raznahan et al., 2010). Recientemente, Reed, Gallagher, Sullivan, Callicott y Green (2017) reportaron diferencias sexuales en tareas de memoria de trabajo. Otra evidencia de la relación entre los esteroides sexuales, la cognición y el funcionamiento cerebral proviene de los estudios sobre las fases del ciclo menstrual, en los que se ha observado que las mujeres obtienen puntajes diferentes en algunas pruebas cognitivas como rotación mental y fluidez verbal de acuerdo con la fase del ciclo en la que se encuentren (Lisofsky, Lindenberger, \& Kühn, 2015).

Estas investigaciones sugieren que las hormonas sexuales influyen en el rendimiento en pruebas cognitivas, y los resultados de la presente investigación muestran evidencia a favor de esta hipótesis, puesto que las participantes del GSOP presentaron menor puntaje en el Cl Verbal, comprensión verbal, memoria de trabajo y fluidez semántica que las del GCT. En concordancia con estos hallazgos, Schattmann y Sherwin, (2007a) observaron que las pacientes con SOP tenían un menor rendimiento en la prueba de fluidez semántica. Por el contrario, Rees et al., (2016) no encontraron evidencia de alteraciones en la prueba de fluidez verbal, sin embargo, se reporta una tendencia ( $p=0.06)$ a un menor rendimiento en la prueba de fluidez semántica en las pacientes con SOP. Son pocos los estudios que han evaluado esta función en pacientes con este diagnóstico, por lo que se sugiere continuar explorándo, por ejemplo, tomando en cuenta el funcionamiento cerebral de la corteza prefrontal y temporal izquierda, las cuales se han relacionado con la ejecución de las pruebas de fluidez verbal semántica y fonológica (Chouiter et al., 2016).

Respecto de los resultados en las escalas ICV e IMT, se observó que las participantes del GSOP obtuvieron un menor puntaje que las del GCT. Hallazgos similares fueron reportados por Rees et al., (2016), quienes encontraron que un grupo de mujeres con diagnóstico de SOP presentó menor rendimiento en las pruebas de dígitos en regresión y progresión, así como de vocabulario y similitudes de la prueba WAIS III. Así mismo, Soleman et al., (2016) utilizaron cuatro versiones de la prueba N-back con diferente grado de dificultad para evaluar memoria de trabajo y no encontraron diferencias en la ejecución entre un grupo de pacientes con diagnóstico de SOP y un grupo de control; sin embargo, reportaron diferencias entre los dos grupos en el funcionamiento cerebral, evaluado mediante resonancia magnética funcional (fMRI), principalmente en las regiones parietal superior e inferior y temporal superior bilaterales, siendo mayor la actividad de estas regiones en el grupo con diagnóstico 
de SOP; y señalan que dicho hallazgo podría significar que las pacientes requerirían más recursos neuronales para obtener un rendimiento en estas tareas similar al del grupo de control.

Al comparar los resultados de los análisis hormonales no se encontraron diferencias significativas entre las participantes del GSOP y del GCT, no obstante, las del GSOP presentaron signos clínicos de hiperandrogenismo (hirsutismo, acné y/o alopecia) y oligo/anovulación. Es posible que el tamaño muestral y la variabilidad individual sean factores que hayan influido en estos resultados. Las diferencias cognitivas encontradas podrían deberse a las condiciones hormonales de las pacientes con diagnóstico de SOP, pero también podrían existir otras variables biológicas que influirían en el rendimiento cognitivo que no se contemplaron en este estudio, por ejemplo, la presencia de insulinorresistencia y otros trastornos metabólicos (Anagnostis et al., 2018; Fenichel, Rougiera, Hieronimus, \& Chevalier, 2017; Rees et al., 2016). Por lo anterior se sugiere continuar investigando la relación de diferentes factores biológicos presentes en el SOP con el funcionamiento cognitivo en estas pacientes.

\section{Conclusiones}

Con base en los resultados de la presente investigación y en estudios previos se observa que las pacientes con diagnóstico de SOP muestran un menor rendimiento en pruebas cognitivas al compararlas con un grupo sin SOP, principalmente en pruebas verbales, lo que podría relacionarse con las alteraciones hormonales propias del Síndrome (manifiestas a través del hiperandrogenismo y la oligo/anovulación). Es posible que estas variaciones hormonales estén ejerciendo un efecto activador a nivel cerebral que se evidencia en la ejecución de pruebas cognitivas; al respecto, existen investigaciones en las que se han evaluado a las pacientes antes y después de tratamiento antiandrogénico y se ha observado un cambio en el rendimiento en pruebas cognitivas (Schattmann \& Sherwin, 2007b) y en el funcionamiento cerebral (Soleman et al., 2016).

No obstante, el tamaño de la muestra y el alcance descriptivo comparativo del presente estudio, se constituyen en limitaciones, por lo que se requiere explorar con mayor detalle las funciones cognitivas de estas pacientes utilizando métodos experimentales, junto con diversas pruebas y técnicas, y estudiar su relación con los diferentes factores biológicos que subyacen a esta patología.

\section{Referencias}

Anagnostis, P., Tarlatzis, B.C., \& Kauffman, R.P. (2018). Polycystic ovarian syndrome (PCOS): Long-term metabolic consequences. Metabolism: Clinical and Experimental, 86, 33-43. doi: https://doi.org/10.1016/j.metabol.2017.09.016

Barnard, L., Balen, A.H., Ferriday, D., Tiplady, B., \& Dye, L. (2007). Cognitive functioning in polycystic ovary syndrome. Psychoneuroendocrinology, 32, 906-914. doi: http:// dx.doi.org/10.1016/j.psyneuen.2007.06.010

Barry, J.A., Parekh, H.S.K., \& Hardiman, P.J. (2013). Visual-spatial cognition in women with polycystic ovarian syndrome: the role of androgens. Human Reproduction, 28(10), 2832-2837. doi: http://dx.doi.org/10.1093/humrep/det335

Bozdag, G., Mumusoglu, S., Zengin, D., Karabulut, E., \& Yildiz, B.O. (2016). The prevalence and phenotypic features of polycystic ovary syndrome: A systematic review and meta-analysis. Human Reproduction, 31(12), 2841-2855. doi: http://dx.doi. org/10.1093/humrep/dew218 
Chouiter, L., Holmberg, J., Manuel, A.L., Colombo, F., Clarke, S., Annoni, J.-M., \& Spierer, L. (2016). Partly segregated cortico-subcortical pathways support phonologic and semantic verbal fluency: A lesion study. Neuroscience, 329, 275-283. doi: http://dx.doi.org/10.1016/j.neuroscience.2016.05.029

Corsi-Cabrera, M., del Río-Portilla, Y., \& Muñoz-Torres, Z. (2007). Sex-steroid dimorfic effects on functional brain organization: Differences in cognition, emotion and anxiolysis. En: M. T. Czerbska (Ed.). Psychoneuroendocrinology Research Trends. New York: Nova Biomedical.

Fenichel, P., Rougiera, C., Hieronimus, S., \& Chevalier, N. (2017). Which origin for polycystic ovaries syndrome: Genetic, environmental or both? Annales d'Endocrinologie, 78, 176-185. doi: http://dx.doi.org/10.1016/j.ando.2017.04.024

Jolliffe, L.T. (1986). Principal component analysis. New York: Springer-Verlag.

Lisofsky, N., Lindenberger, U., \& Kühn, S. (2015). Amygdala/hippocampal activation during the menstrual cycle: Evidence for lateralization of effects across different tasks. Neuropsychologia, 67, 55-62. doi: http://dx.doi.org/10.1016/j.neuropsychologia.2014.12.005

Martino, P. L., Bonet, J.L., \& De Bortoli, M.A. (2015). Síndrome de ovario poliquístico: una revisión de sus correlatos patológicos en el ánimo y en el rendimiento cognitivo. Revista Chilena de Neuropsicología, 10(1), 38-43. doi: http://dx.doi.org/10.5839/ renp.2015.10.01.08

Moran, C., Tena, G., Moran, S., Ruiz, P., Reyna, R., \& Duque, X. (2010). Prevalence of Polycystic Ovary Syndrome and Related Disorders in Mexican Women. Gynecologic and Obstetric Investigation, 69(4), 274-280. doi: http://dx.doi.org/10.1159/000277640

Ostrosky-Solís, F., Gómez, M. E., Matute, E., Rosselli, M., Ardila, A., \& Pineda, D. (2003). NEUROPSI ATENCIÓN Y MEMORIA 6 a 85 años. México: American Book Store \& Teletón.

Ostrosky-Solís, F., Gómez Pérez, M. E., Matute, E., Rosselli, M., Ardila, A., \& Pineda, D. (2007). NEUROPSI ATTENTION AND MEMORY: A Neuropsychological test battery in Spanish with norms by age and educational level. Applied Neuropsychology, 14(3), 156-170. doi: http://dx.doi.org/10.1080/09084280701508655

Raznahan, A., Lee, Y., Stidd, R., Long, R., Greenstein, D., Clasen, L., ... Giedd, J.N. (2010). Longitudinally mapping the influence of sex and androgen signaling on the dynamics of human cortical maturation in adolescence. Proceedings of the National Academy of Sciences, 107(39), 16988-16993. doi: http://dx.doi.org/10.1073/ pnas.1006025107

Reed, J.L., Gallagher, N.M., Sullivan, M., Callicott, J.H., \& Green, A.E. (2017). Sex differences in verbal working memory performance emerge at very high loads of common neuroimaging tasks. Brain and Cognition, 113, 56-64. doi: http://dx.doi. org/10.1016/j.bandc.2017.01.001

Rees, D. A., Udiawar, M., Berlot, R., Jones, D. K., \& O'Sullivan, M. J. (2016). White matter microstructure and cognitive function in young women with polycystic ovary syndrome. The Journal of Clinical Endocrinology and Metabolism, 101(1), 314-323. doi: http://dx.doi.org/10.1210/jc.2015-2318

Rotterdam ESHRE/ASRM-Sponsored PCOS Consensus Workshop Group (2004). Revised 2003 consensus on diagnostic criteria and long-term health risks related to polycystic ovary syndrome (PCOS). Human Reproduction, 19, 41-47. doi: http:// dx.doi.org/10.1016/i.fertnstert.2003.10.004

Schattmann, L., \& Sherwin, B.B. (2007a). Testosterone levels and cognitive functioning in women with polycystic ovary syndrome and in healthy young women. Hormones and Behavior, 51(5), 587-596. doi: http://dx.doi.org/10.1016/j.yhbeh.2007.02.007

Schattmann, L., \& Sherwin, B.B. (2007b). Effects of the pharmacologic manipulation of testosterone on cognitive functioning in women with polycystic ovary syn- 
drome: A randomized, placebo-controlled treatment study. Hormones and Behavior, 51(5), 579-586. doi: http://dx.doi.org/10.1016/j.yhbeh.2007.02.002

Scheuringer, A., \& Pletzer, B. (2017). Sex differences and menstrual cycle dependent changes in cognitive strategies during spatial navigation and verbal fluency. Frontiers in Psychology, 8(381), 1-12. doi: https://doi.org/10.3389/fpsyg.2017.00381

Soleman, R.S., Kreukels, B.P.C., Veltman, D.J., Cohen-Kettenis, P.T., Hompes, P.G.A., Drent, M.L., \& Lambalk, C.B. (2016). Does polycystic ovary sindrome affect cognition? A functional magnetic resonance imaging study exploring working memory. Fertility and Sterility, 105(5), 1314-1321. doi: http://dx.doi.org/10.1016/j.fertnstert.2016.01.034

Voyer, D. (2011). Time limits and gender differences on paper-and-pencil tests of mental rotation: a meta-analysis. Psychonomic Bulletin \& Review, 18(2), 267-277. doi: https://doi.org/10.3758/s13423-010-0042-0

Wechsler, D. (1997/2003a). Escala Wechsler de Inteligencia para Adultos (WAIS-III). (3ra. ed.) México: Manual Moderno.

Wechsler, D. (1997/2003b). Escala Wechsler de Inteligencia para Adultos (WAIS-III) Manual técnico. (3ra. ed.) México: Manual Moderno. 